\title{
X-ray Emission from Old and Intermediate Age Brown Dwarfs
}

\author{
Beate Stelzer \& Ralph Neuhäuser \\ Max-Planck-Institut für extraterrestrische Physik, Postfach 1312, \\ D-85741 Garching, Germany
}

\begin{abstract}
We report on two recent XMM-Newton observations of Brown Dwarfs in the Pleiades cluster and in the field aiming to constrain the age dependence of X-ray emission from substellar objects.
\end{abstract}

\section{Introduction}

The standard picture of solar-type magnetic activity is expected to break down for very-low mass stars: being fully convective throughout the interior they lack the interface between radiative core and convective envelope in which the solartype $\alpha \Omega$-dynamo is thought to reside. In spite of these theoretical predictions $\mathrm{X}$-ray and $\mathrm{H} \alpha$ activity has been observed on stars with masses below the fully convective boundary, corresponding to spectral type M3 (Fleming et al. 1995, Gizis et al. 2000). Recent $\mathrm{H} \alpha$ observations of ultracool field dwarfs indicate, however, a decline of activity setting in near the substellar limit at spectral type M9 (Mohanty \& Basri 2002, see also Basri this volume).

While there is a substantial data base on chromospheric activity, only few X-ray observations of very low-mass (VLM) field dwarfs have been performed so far. Virtually all of the X-ray emitting field dwarfs with spectral type later than $\sim$ M7 have been detected only during a temporary outburst, with quiescent emission below the detection threshold (see Sect. 3). Whether this is due to a lack of sensitivity of the respective observations, or whether these objects indeed are X-ray quiet can now be tested with a new generation of X-ray instruments onboard XMM-Newton and Chandra.

X-ray observations with ROSAT have shown that young Brown Dwarfs (BDs) are more readily detected than older ones, as they show higher levels of activity (Neuhäuser et al. 1999). Due to the absence of an internal energy source the evolution of BDs goes along with a decrease of effective temperature. The accompanying drop of the ionization fraction may prevent coupling of the gas to the magnetic field, thus shutting off activity. Probing the relation between activity and the evolution of atmospheric conditions requires high-sensitivity X-ray observations of VLM stars and BDs at different ages.

\section{Observations}

In order to examine the dependence of X-ray emission on age and/or effective temperature, we observed two BDs in different evolutionary stages: (a) the 


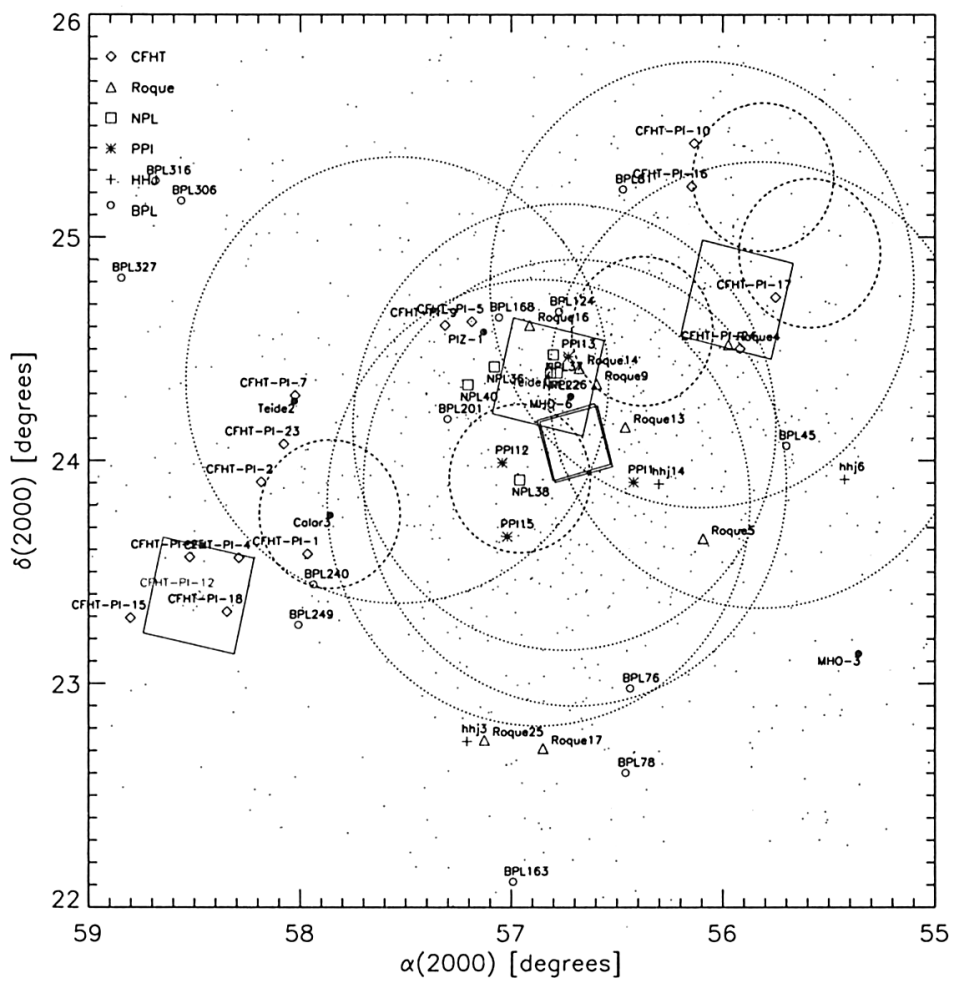

Figure 1. Sky map of the Pleiades region with position of X-ray pointings by ROSAT (small circles = HRI, large circles = PSPC), Chandra (small squares), and XMM-Newton (large squares); dots are Pleiades members.

$\sim 100$ Myr old BD CFHT-Pl 12 in the Pleiades cluster, and (b) Denis J12281547 which is a BD binary in the field at an age of $\sim 500 \mathrm{Myr}$.

The Pleiades cluster has been extensively monitored with past and present day X-ray instrumentation (see sky map in Fig. 1 presenting all X-ray observations centered on the Pleiades since the ROSAT mission). None of the earlier observations was deep enough to reach into the substellar regime. In particular the limiting sensitivity of pointed ROSAT observations in the Pleiades region is $\lg L_{\mathrm{x}} \sim 28.5 \ldots 29.0 \mathrm{erg} / \mathrm{s}$ (Stelzer \& Neuhäuser 2001). Two long exposures with Chandra included no BDs in the field (Krishnamurthi et al. 2000, Daniel et al. 2002). Here, we discuss one of the three XMM-Newton pointings performed up to now. As seen from Fig. 1 CFHT-Pl 12 is located in a region of the cluster which is widely unexplored in X-rays so far. CFHT-Pl12 is the optically brightest of the BD candidates in the Pleiades, and its position above the mainsequence in the color-magnitude diagram indicates it could be a binary (Bouvier et al. 1998). Unresolved binaries should have a higher probability of detection, 
and therefore also Denis J1228-1547, a BD binary with $\sim 0.3^{\prime \prime}$ separation, is a favorable target for X-ray studies.

\section{Results}

The data were analysed using the standard $X M M-$ Newton SAS, version 5.3.0. Strong background flaring restricted the useful exposure time to $\sim 8.5 \mathrm{ksec}$ for CFHT-Pl 12 and $\sim 6 \mathrm{ksec}$ for Denis J1228-1547. Source detection was performed in three energy bands in the range from 0.3 to $5.0 \mathrm{keV}$ using a combination of local, map, and maximum likelihood detection mechanism. Neither CFHT-Pl12 nor Denis J1228-1547 are detected at a $M L$ detection threshold of 10. Upper limits to the source flux derived under the assumption of a one-temperature, $1 \mathrm{keV}$ Raymond-Smith spectrum plus photo-absorption are listed in Table 1. To facilitate comparison with earlier X-ray observations of VLM objects, the luminosities were converted to the $0.1-2.4 \mathrm{keV}$ band covered by ROSAT.

Table 1. X-ray properties of two BDs observed with XMM-Newton.*

\begin{tabular}{lrrrrrr}
\hline Instr. & Cts & $\begin{array}{c}\text { Expo } \\
{[\mathrm{s}]}\end{array}$ & $\begin{array}{c}F_{\mathrm{x}} \\
{\left[\mathrm{erg} / \mathrm{s} / \mathrm{cm}^{2}\right]}\end{array}$ & $\begin{array}{c}\lg L_{\mathrm{x}} \\
{[\mathrm{erg} / \mathrm{s}]}\end{array}$ & $\lg \left(\frac{L_{\mathrm{x}}}{L_{\mathrm{bol}}}\right)$ & $\begin{array}{r}\lg L_{\mathrm{x}} \\
(\mathrm{RASS})\end{array}$ \\
\hline CFHT-P112 \\
pn & 14 & 8593 & $<4.1 \cdot 10^{-15}$ & $<27.28$ & $<-3.48$ & $<29.3$ \\
\hline MOS 1+2 & 29 & 34481 & $<3.5 \cdot 10^{-15}$ & $<27.76$ & $<-3.00$ & \\
\hline \hline Dn & 20 & 5889 & $<4.0 \cdot 10^{-15}$ & $<26.15$ & $<-3.14$ & $<27.3$ \\
\hline MOS 1+2 & 15 & 15596 & $<5.8 \cdot 10^{-15}$ & $<26.30$ & $<-2.99$ & \\
\hline \hline
\end{tabular}

${ }^{*}$ Counts are measured in the broad band of EPIC $(0.3-5 \mathrm{keV})$, but X-ray flux and luminosity and their $3 \sigma$ confidence upper limits apply to the ROSAT band of $0.1-2.4 \mathrm{keV}$; last column are data from RASS.

In Fig. 2 the newly derived upper limit for Denis J1228-1547 is compared to X-ray observations of other field dwarfs: M-stars from the Catalog of Nearby Stars (CNS; Gliese \& Jahreiss 1991) detected in the ROSAT All-Sky Survey (=RASS; data from Hünsch et al. 1999), and field dwarfs later than M6 observed with ROSAT in pointed observations and/or with Chandra (see Neuhäuser et al. 1999, Fleming et al. 2000, Rutledge et al. 2000, Schmitt \& Liefke 2002). The XMM-Newton observation of Denis J1228-1547 presented here is the first attempt to extend the study of X-ray activity into spectral class L. The upper limit derived from the RASS is improved by $\sim 1$ order of magnitude for this object despite the considerable loss of observing time (due to high background). Note, that at the time of target selection only three BDs in the field had been discovered. In the near future, longer XMM-Newton observations of the nearby early L-type dwarfs identified since then are likely to provide faint detections or meaningful upper limits populating the lower right of Fig. 2. 


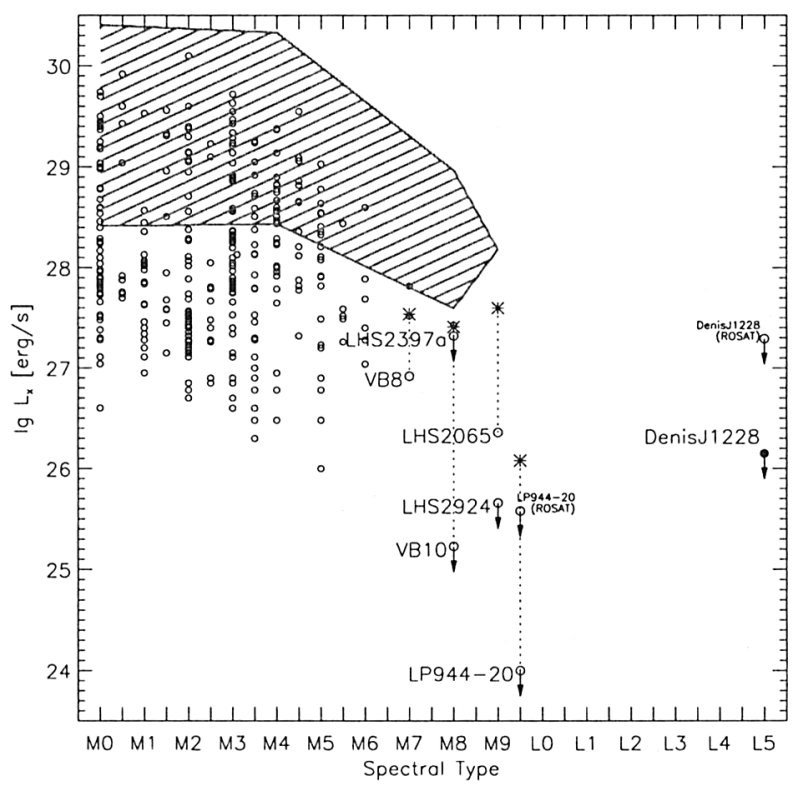

Figure 2. $\quad \lg L_{\mathrm{x}}$ over spectral type for field M and L dwarfs. Asterisks denote flares, arrows denote upper limits for non-detections. For Denis J1228-1547 and LP 944-20 both the ROSAT and the XMM-Newton or Chandra measurements are given. The typical region occupied by younger objects of the same spectral type found in star forming regions (data from Mokler \& Stelźer 2002) is shown as hatched area.

\section{References}

Bouvier J., Stauffer J. R., Martín E. L., et al., 1998, A\&A 336, 490

Daniel K., Linksy J. L., Gagné M., 2002, astro-ph/0204131

Fleming T. A., Schmitt J. H. M. M., Giampapa M. S., 1995, ApJ 450, 401

Fleming T. A., Giampapa M. S., Schmitt J. H. M. M., 2000, ApJ 533, 372

Gizis J. E., Monet D. G., Reid I. N., et al., 2000, ApJ 120, 1085

Gliese W. \& Jahreiss H., 1991, Prelim. version of the Third Catalogue of Nearby Stars, NASA/Astronomical Data Center, GSFC, Greenbelt, Maryland

Hünsch M., Schmitt J. H. M. M., Sterzik M. F., et al. 1999, A\&AS 135, 319

Krishnamurthi A., Reynolds C. S., Linsky J. L., et al., 2001, AJ 121, 337

Mohanty S. \& Basri G., 2002, astro-ph/0201455

Mokler F. \& Stelzer B., 2002, A\&A in press

Neuhäuser R., Briceño C., Comerón F., et al., 1999, A\&A 343, 883

Rutledge R. E., Basri G., Martín E. L., Bildsten L., 2000, ApJ 538, L141

Schmitt J. H. M. M. \& Liefke C., 2002, A\&A 382, L9

Stelzer B. \& Neuhäuser R., 2001, A\&A 377, 538 